\title{
Type II supernovae as probes of environment metallicity: observations of host $\mathrm{H}$ II regions (Corrigendum)
}

J. P. Anderson ${ }^{1}$, C. P. Gutiérrez ${ }^{1,2,3}$, L. Dessart ${ }^{4}$, M. Hamuy ${ }^{3,2}$, L. Galbany ${ }^{2,3}$, N. I. Morrell ${ }^{5}$, M. D. Stritzinger ${ }^{6}$, M. M. Phillips ${ }^{5}$, G. Folatelli ${ }^{7}$, H. M. J. Boffin ${ }^{1}$, T. de Jaeger ${ }^{2,3}$, H. Kuncarayakti ${ }^{2,3}$, and J. L. Prieto ${ }^{8,2}$

1 European Southern Observatory, Alonso de Córdova 3107, Casilla 19, Santiago, Chile e-mail: janderso@eso.org

2 Millennium Institute of Astrophysics, Casilla 36-D, Santiago, Chile

3 Departamento de Astronomía, Universidad de Chile, Camino El Observatorio 1515, Las Condes, Santiago, Chile

${ }^{4}$ Laboratoire Lagrange, Université Côte d'Azur, Observatoire de la Côte d'Azur, CNRS, Boulevard de l'Observatoire, CS 34229 , 06304 Nice Cedex 4, France

5 Carnegie Observatories, Las Campanas Observatory, Casilla 601, La Serena, Chile

6 Department of Physics and Astronomy, Aarhus University, Ny Munkegade 120, 8000 Aarhus C, Denmark

7 Instituto de Astrofísica de La Plata, Facultad de Ciencias Astronómicas y Geofísicas, Universidad Nacional de La Plata, CONICET, Paseo del Bosque S/N, B1900FWA, La Plata, Argentina

8 Núcleo de Astronomía de la Facultad de Ingeniería, Universidad Diego Portales, Av. Ejército 441, Santiago, Chile

\section{A\&A 589, A110 (2016), DOI: 10.1051/0004-6361/201527691}

Key words. supernovae: general - HII regions - galaxies: abundances - errata, addenda

An error occurred during the production process. The title of the article in the published version was "Type II supernovae as probes of environment metallicity: observations of host He II regions". However, this should be: "Type II supernovae as probes of environment metallicity: observations of host H II regions". 